\title{
Use of Methadone for Opioid Weaning in Children: Prescribing Practices and Trends
}

\author{
Kazim Giby, Régis Vaillancourt, Nisha Varughese, Christina Vadeboncoeur, and Annie Pouliot
}

\section{INTRODUCTION}

C ritically ill children are routinely treated with opioids to relieve pain, produce sedation, increase cooperation, and reduce metabolic demands. ${ }^{1}$ However, prolonged exposure to opioids followed by abrupt cessation frequently leads to opioid withdrawal syndrome. ${ }^{2,3}$ This syndrome is present in $35 \%$ to $57 \%$ of children who have received fentanyl by continuous infusion. ${ }^{4-6}$ It is characterized by a hyperadrenergic response following the abrupt reduction in opioid dosage, with clinical symptoms that include irritability, tremors, twitching, gastrointestinal disturbances, hypertension, and fever. ${ }^{4-6}$ The dose and duration of opioid use influence the development of opioid withdrawal symptoms. ${ }^{4,5,7}$ However, proper pharmacologic management of withdrawal is still controversial, and there is little evidence upon which to base recommendations regarding prevention, assessment, and management of withdrawal in critically ill children. ${ }^{8}$

Opioid weaning protocols aim to gradually decrease plasma concentrations of the drug to prevent symptoms of opioid withdrawal syndrome., ${ }^{910}$ Oral morphine, methadone, and clonidine and transdermal fentanyl have all been used in opioid weaning programs, but methadone is often the most suitable, because of the convenience of oral dosing and the long half-life. ${ }^{11}$

Currently, there is great variability among recommended methadone weaning protocols to prevent withdrawal symptoms in the pediatric population. ${ }^{12-14}$ Some factors that may influence the methadone dosing schedule are initial diagnosis; duration of opioid therapy; patient's age, developmental stage, and sex; and interacting concomitant medications. ${ }^{910,12-14}$

At the time of the current study, methadone prescription protocols at the authors' institution were designed on a case-bycase basis. The authors believed that standardization of opioid weaning protocols would result in safer dosing schedules, fewer cases of withdrawal symptoms, and shorter hospital stays. ${ }^{15}$

The objectives of this study were to describe weaning practices in a hospital where weaning was done on a case-by- case basis, without standardized protocols; to determine whether there were common patterns for methadone-weaning practices shown to be effective; and to quantify and characterize any patterns found.

\section{METHODS}

This retrospective study involved chart review for patients who received methadone for weaning from opioid therapy at the Children's Hospital of Eastern Ontario (CHEO) between December 2006 and May 2012. CHEO is a tertiary care pediatric teaching hospital located in Ottawa, Ontario, with a catchment area that includes eastern Ontario, western Quebec, and the Baffin region. This study was approved by the CHEO Research Ethics Board.

The CHEO pharmacy's administrative software was used to identify all patients associated with any inpatient service within the hospital for whom methadone was prescribed during the 5.5-year study period. Inclusion criteria were age 18 years or younger, receipt of opioid (fentanyl, morphine, hydromorphone) by IV infusion within 7 days before first dose of methadone, receipt of methadone for more than 3 consecutive days, and completion of weaning in the hospital. Reasons for unsuccessful or incomplete weaning included death, transfer or discharge from CHEO, or administration of opioids within 5 days of the last dose of methadone. Data extracted from the patients' charts were age, weight, diagnosis on admission, duration of hospital stay, opioids received, methadone dosing schedule, concomitant administration of sedatives during methadone administration, and dates of endotracheal intubation.

To determine total daily doses and duration of weaning, the methadone and opioid dosing schedule was divided into distinct 24-h segments for each patient, with day 1 of the methadone wean being defined as the 24-h period following the first dose of methadone and day $(-1)$ being defined as the $24 \mathrm{~h}$ preceding the first dose of methadone. Opioid dosing was recorded for a maximum of 7 days $(168 \mathrm{~h})$ before the first dose of methadone and throughout the weaning period. Fentanyl 
and hydromorphone doses were converted to equivalents of IV morphine to allow comparison of opioid dosing between patients. In cases where different opioids were used on the same day, opioid doses were converted to equivalents of morphine, and the sum of doses was used as the total daily dose of opioids. Opioid conversion ratios were obtained from the 2009/2010 formulary of the Hospital for Sick Children, Toronto, Ontario ${ }^{16}$ (see Table 1).

\section{Data Analysis}

Large-scale quantitative data analysis was conducted using SPSS version 20 software (IBM, Armonk, New York). Measured variables were evaluated with descriptive statistics. Continuous variables with normal distribution are presented as means \pm standard deviations; variables with a non-Gaussian distribution are presented as medians and ranges. To determine correlation between variables, a Pearson correlation model was implemented, and significance was determined using a 2-tailed Student $t$ test.

Once the data were obtained, the total daily doses of methadone and opioids were standardized to units of milligrams per kilogram per day, which were then plotted against weaning period (days).

A review panel consisting of 2 pharmacists (R.V., N.V.) and 1 physician (C.V.) was created. The panel's objective was to qualitatively identify any consistent, recognizable patterns common to the patients' weaning schedules. All of the panel's decisions were based on consensus. Methadone doses were then converted from milligrams per kilogram per day to percentage of maximum methadone dose, to allow comparison of daily dose reductions between patients. These results were used for linear regression analysis, which showed significant linear consistency over time. The regression results were used to generate criteria identifying different weaning groups. The methadone dose for any given phase of weaning was calculated with the following formula:

Daily methadone dose during phase $=$

( $\sum$ methadone doses during phase)/(duration of phase)

All daily methadone doses during the tapering phase were converted to percent of maximum methadone tapering dose, which was calculated for each patient according to the following formula:

$\%$ maximum methadone tapering dose =

(methadone tapering dose/maximum tapering dose for patient X) $\times 100$

Although the initial intention was to assess withdrawal using the Withdrawal Assessment Tool (WAT-1), ${ }^{17}$ in practice

\section{Table 1. Opioid Conversion Table*}

\begin{tabular}{lcc} 
& \multicolumn{1}{c}{ Morphine-Equivalent Analgesic Dose (mg) } \\
\cline { 2 - 3 } Drug & IM or IV & PO \\
\hline Morphine & 1 & 3 \\
Fentanyl & 0.01 & NA \\
Hydromorphone & 0.15 & 0.45 \\
\hline $\mathrm{IM}=$ intramuscular, IV = intravenous, NA = not applicable, \\
PO = by mouth. \\
*Used to standardize opioid dosing. The constants presented \\
here were employed to convert opioids of different strengths \\
to equivalent morphine doses. Adapted from "Opioid Equianal- \\
gesic Conversion Chart", Hospital for Sick Children formulary, \\
2009/2010. ${ }^{16}$
\end{tabular}

Table 2. Demographic Characteristics of 30 Patients Included in the Study

\begin{tabular}{lc}
\multicolumn{1}{c}{ Characteristic } & Value \\
\hline Age (years), ${ }^{*}$ median (IQR) & $3.46(0.08-16.4)$ \\
Weight (kg), ${ }^{*}$ median (IQR) & $7.6(2.9-60)$ \\
Sex, no. (\%) & $17(57)$ \\
Males & $13(43)$ \\
Females & 13 is \\
*Age and weight were not normally distributed in this sample \\
(Shapiro-Wilk test for significance, $p<0.001$ for both age and \\
weight).
\end{tabular}

this was not possible, because the WAT-1 tool had been implemented only recently at the study institution and only $20 \%$ of the patients in the study cohort had been assessed with this tool. Before adoption of the objective WAT-1 tool at CHEO, only subjective assessment of withdrawal events had been performed, and inclusion of these subjective assessments might have complicated interpretation of results. Therefore, withdrawal events were not included in the current analysis.

\section{RESULTS}

\section{Demographic Characteristics}

A total of 73 patients received methadone at the study hospital in the 5.5-year study period. Of these, 30 (41\%) met the inclusion criteria for this study. Of the 43 patients excluded from the study, 1 patient was over the age of 18 years, 3 had not received an opioid infusion 7 days before methadone weaning, 7 had not received methadone for more than 3 consecutive days, and 32 had incomplete weaning. Patient demographic characteristics are listed in Table 2 and diagnoses on admission in Table 3.

\section{Structure of Weaning Schedule}

The mean duration of methadone weaning, from first to final dose, was $19.8 \pm 9.5$ days (range 5-40 days); the duration of methadone weaning was normally distributed (Shapiro Wilk 
Table 3. Diagnosis at Time of Admission

\begin{tabular}{|c|c|}
\hline Patient no. & Admission diagnosis \\
\hline 1 & Apneic episodes \\
\hline 2 & Second- and third-degree burns \\
\hline 3 & Severe burns \\
\hline 4 & First- and third-degree burns \\
\hline 5 & $\begin{array}{l}\text { Repair of tetralogy of Fallot and atrioventricular } \\
\text { septal defect }\end{array}$ \\
\hline 6 & $\begin{array}{l}\text { Vomiting with increased respiratory effect, } \\
\text { systemic lymphangiomatosis }\end{array}$ \\
\hline 7 & $\begin{array}{l}\text { Acquired brain injury secondary to right epidural } \\
\text { hematoma }\end{array}$ \\
\hline 8 & Altered level of consciousness, psychosis \\
\hline 9 & $\begin{array}{l}\text { Extreme prematurity, very low birth weight, } \\
\text { necrotizing enterocolitis, sepsis, pulmonary } \\
\text { hemorrhage }\end{array}$ \\
\hline 10 & Repair of tetralogy of Fallot \\
\hline 11 & Bilateral choanal atresia \\
\hline 12 & Tracheoesophageal fistula \\
\hline 13 & $\begin{array}{l}\text { Conversion disorder, difficulty eating because } \\
\text { of vomiting, loss of consciousness, inability to } \\
\text { keep fluids down }\end{array}$ \\
\hline 14 & Herpes simplex virus encephalitis, seizure disorder \\
\hline 15 & $\begin{array}{l}\text { Severe obstructive sleep apnea, pneumonia, } \\
\text { Clostridium difficile infection }\end{array}$ \\
\hline 16 & Tracheoesophageal fistula \\
\hline 17 & Trisomy 21, repair of atrioventricular septal defect \\
\hline 18 & Respiratory failure \\
\hline 19 & $\begin{array}{l}\text { Relapsing acute lymphoblastic leukemia with } \\
\text { cardiac dysfunction }\end{array}$ \\
\hline 20 & Multiple trauma \\
\hline 21 & Asthma \\
\hline 22 & $\begin{array}{l}\text { Intracranial hemorrhage secondary to } \\
\text { arteriovenous fistula }\end{array}$ \\
\hline 23 & Congenital heart disease \\
\hline 24 & Pneumonia \\
\hline 25 & Septic shock \\
\hline 26 & Repair of bilateral inguinal hernia \\
\hline 27 & Necrotizing enterocolitis \\
\hline 28 & Tricuspid atresia \\
\hline 29 & $\begin{array}{l}\text { Right medullary-cerebral infarct secondary to } \\
\text { vertebral artery dissection }\end{array}$ \\
\hline 30 & Repair of atrioventricular septal defect \\
\hline
\end{tabular}

test of significance, $p=0.12$, data not shown). Preliminary analysis of each patient's dosing schedule followed by linear regression analysis revealed 4 distinct, consistent phases of methadone weaning. These 4 phases were used to compare the heterogeneous dosing schedules:

- Overlap phase: concomitant administration of opioids and methadone

- Loading phase: short period (about 2 days) near the beginning of weaning, during which very high doses (about 2 times the average maintenance dose) of methadone are administered to bring plasma levels of methadone to the therapeutic range

- Maintenance phase: characterized by stable dosing schedule that lasts until the patient is deemed ready for tapering

- Tapering phase: characterized by constant decrease in total daily dose of methadone until completion of weaning

The duration and daily dose for each phase are presented in Table 4. For $14(47 \%)$ of the patients, all 4 phases were observed. For another 13 patients (43\%), 3 identifiable phases were observed, and for the remaining 3 patients (10\%), only 2 phases were observed. The terms "methadone overlap dose", "methadone loading dose", and "methadone maintenance dose" refer to the median total daily dose during the overlap, loading, and maintenance phases, respectively. The median total daily dose during the tapering phase was not calculated because this phase is characterized by recurring dose reductions.

\section{Rate of Dose Reduction for Successful Weaning}

Given that for most patients the tapering phase consisted of repeated daily dose reductions at a constant rate, simple linear regression was applied for this phase. All daily methadone doses during the tapering phase were converted to percent of the maximum methadone tapering dose, calculated for each patient according to the formula presented in the Methods section.

\section{Table 4. Median Duration and Daily Dose for 4 Phases of Methadone Weaning Schedule*}

\begin{tabular}{lcccc} 
Phase & $\begin{array}{c}\text { Duration (days), } \\
\text { median (range) }\end{array}$ & $\begin{array}{c}\text { Daily methadone dose } \\
(\mathbf{m g} / \mathbf{k g}),\end{array}$ \\
\hline Opioid overlap $(n=26)$ & 2.0 & $(1-18)$ & 0.44 & $(0.11-1.35)$ \\
Loading $(n=19)$ & 2.0 & $(1-4)$ & 0.50 & $(0.08-2.59)$ \\
Maintenance $(n=26)$ & 2.5 & $(1-17)$ & 0.27 & $(0.03-1.73)$ \\
Tapering $(n=30)$ & 11 & $(4-30)$ & Not calculated \\
\hline *Data for duration of all 4 phases had a non-Gaussian distribution; therefore, medians were \\
calculated. \\
†Not calculated because this phase was characterized by recurring dose reductions.
\end{tabular}


Table 5. Grouping Criteria and Linear Regression Analysis for Short-, Medium-, and Long-Duration Tapering

\begin{tabular}{|c|c|c|c|}
\hline \multirow[b]{2}{*}{ Characteristic } & \multicolumn{3}{|c|}{ Group } \\
\hline & Short Duration & Medium Duration & Long Duration \\
\hline $\begin{array}{l}\text { Length of tapering phase } \\
\text { (days), mean } \pm \text { SD }\end{array}$ & $5 \pm 2$ & $10 \pm 2$ & $15 \pm 2^{*}$ \\
\hline Sample size & 8 & 9 & 13 \\
\hline Regression slope (\%/day) & -15.14 & -8.83 & -4.11 \\
\hline$R^{2}$ value & 0.79 & 0.83 & 0.68 \\
\hline $\begin{array}{l}\text { Morphine-equivalent dose } \\
\text { on day before starting } \\
\text { methadone (mg/kg per day), } \\
\text { mean } \pm \text { SD }\end{array}$ & $2.91 \pm 3.94$ & $5.27 \pm 5.67$ & $3.92 \pm 3.00$ \\
\hline $\begin{array}{l}\text { Total daily dose before } \\
\text { weaning protocol (mg) }\end{array}$ & 3.20 & 5.62 & 5.24 \\
\hline
\end{tabular}

Following linear regression, the best-fit line for all data points $(n=339)$ had a slope of $4.25 \%$ /day, with an $R^{2}$ value of 0.595 (data not shown). The fastest wean (in terms of percent reduction per day) was $25 \% /$ day, and the slowest was $2.3 \% /$ day.

Patients were then subdivided into 3 groups according to duration of weaning (short, medium, long; criteria presented in Table 5). Figure 1 and Table 5 demonstrate a clear division among the groups according to speed of tapering. One patient, who had congenital heart defects, underwent an exceedingly long tapering phase (30 days) and was included in the longduration weaning group, even though duration of this patient's tapering phase was longer than the criterion for this group $(15 \pm 2$ days). There were no observable differences in age, weight, sex, opioid dosing, or mechanical ventilation status among the 3 groups; however, there were significant differences in dose reduction per day and duration of the tapering phase $(p=0.01)$.

Not surprisingly, for patients with short weaning schedules, the previous total daily dose of morphine equivalents was significantly lower (median $3.20 \mathrm{mg} / \mathrm{kg}$ per day) than for those in the medium-duration weaning group (median $5.62 \mathrm{mg} / \mathrm{kg}$ per day) or the long-duration weaning group (median 5.24 $\mathrm{mg} / \mathrm{kg}$ per day).

\section{Methadone and Opioid Dosing}

Opioid dosing on the day before weaning (in milligrams morphine equivalents per kilogram per day) showed a significant positive correlation with methadone maintenance dosing $(p=0.05)$. The ratio of methadone maintenance doses to prior opioid doses was $0.35 \pm 0.92(n=26)$. The methadone loading dose was strongly correlated with the methadone maintenance dose in patients who had both these phases $(p=0.01 ; n=18)$.
The ratio of methadone loading dose to methadone maintenance dose was $2.10 \pm 0.97$. In addition, the methadone loading dose had a significant inverse correlation with patient's weight $(p=0.015)$ and age $(p=0.025)$, whereas the methadone maintenance dose was not affected by weight $(p=0.06)$ or age $(p=0.07)$. Sex had no significant effect on methadone or opioid dosing in this sample.

\section{Use of Concomitant Medications during Methadone Weaning}

Twenty-nine (97\%) of the 30 patients were taking at least one other medication known to affect opioid or methadone dosing during methadone weaning (see Table 6 for the most common of these medications). The mean number of concomitant medications was $4.6 \pm 2.1$. No relationship was observed between the number or types of medication taken and the opioid and methadone dosing schedules.

\section{Mechanical Ventilation and Methadone Weaning Schedule}

Twenty (67\%) of the 30 patients underwent mechanical ventilation during the $168 \mathrm{~h}$ before the first dose of methadone. Of these 20 patients, 10 were still intubated during receipt of methadone, whereas the other 10 were extubated before methadone weaning was started. For the 10 patients who received methadone concurrent with mechanical ventilation, the number of days of intubation during methadone weaning was $9.7 \pm 10$ (range 1-26 days). The number of days of intubation during methadone weaning was strongly and positively correlated with the duration of methadone weaning ( $p=0.03)$, the duration of the overlap phase $(p<0.001)$, and the duration of the maintenance phase $(p=0.022)$. However, there were no significant differences in overall weaning times or 


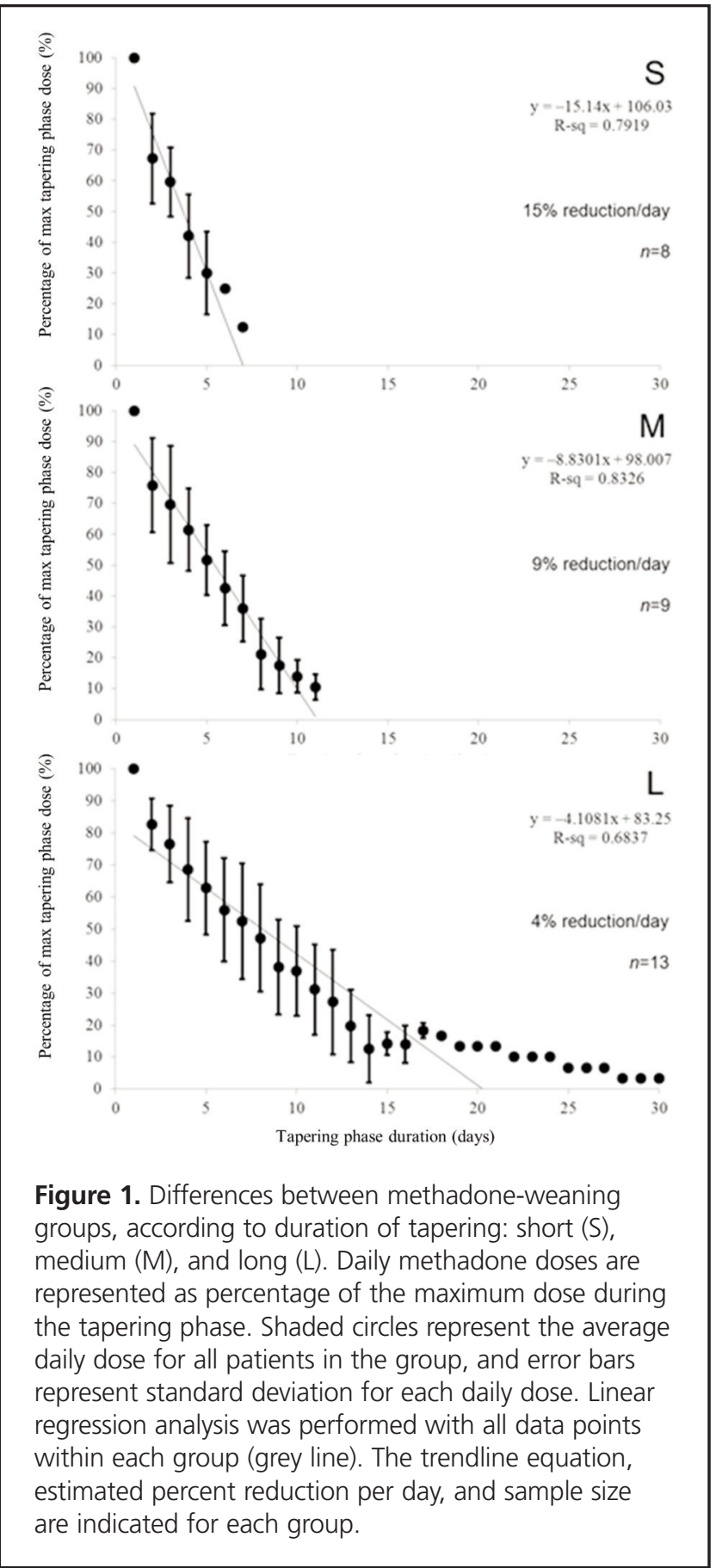

duration of the methadone weaning phases in nonintubated patients.

Not surprisingly, methadone and opioid dosing differed between intubated and nonintubated individuals, with the daily methadone overlap doses being $0.66 \mathrm{mg} / \mathrm{kg}$ and 0.40 $\mathrm{mg} / \mathrm{kg}$, respectively. In addition, the opioid dose on the day before methadone weaning, in morphine equivalents, was significantly higher among intubated patients than nonintubated patients ( 4.96 versus $2.24 \mathrm{mg} / \mathrm{kg}$ per day; $p=0.05$ ).
Table 6. Most Common Sedative and Nonsedative Medications Used during Methadone Weaning

\begin{tabular}{lrr} 
Drug & No. (\%) of patients \\
\hline Clonidine & 23 & $(77)$ \\
Melatonin & 23 & $(77)$ \\
Benzodiazepines & 21 & $(70)$ \\
Lorazepam & 19 & $(63)$ \\
Midazolam & 18 & $(60)$ \\
Diazepam & 1 & $(3)$ \\
Chloral hydrate & 20 & $(67)$ \\
Diphenhydramine & 20 & $(67)$ \\
Dexmedetomidine & 5 & $(17)$ \\
Dexamethasone & 5 & $(17)$ \\
Propofol & 3 & $(10)$ \\
Chlorpromazine & 2 & $(7)$ \\
\hline & &
\end{tabular}

The literature presents several approaches to prevent opiate withdrawal symptoms. Although patients can be weaned slowly from continuous infusions, a more convenient alternative is to substitute opioids with methadone, a longacting synthetic opioid with good oral bioavailability. ${ }^{18}$ Methadone has the longest half-life of any of the commonly available opiates (19 h in children, $35 \mathrm{~h}$ in adults), and $70 \%$ to $100 \%$ oral bioavailability, making it the preferred drug for narcotic withdrawal for infants and children. ${ }^{18,19}$ The oral bioavailability of methadone allows for conversion from the IV to the oral route of administration, and its long half-life allows for extended dosing intervals (e.g., every $24 \mathrm{~h}$ ). Methadone has been used for adults with chronic opiate dependency, and pharmacokinetic information for the use of methadone in the pediatric population is now available. . $021^{21}$

A number of prospective studies have examined the effects of duration, ${ }^{2,22}$ dosing range, ${ }^{3}$ and standardization of protocol ${ }^{15}$ for methadone weaning. Retrospective studies, which are less common, ${ }^{11,23}$ have focused on evaluating the effectiveness and implementation of standardized protocols.

The current analysis of methadone weaning schedules for 30 patients revealed 4 distinct phases of methadone weaning: overlap, loading, maintenance, and tapering. These phases were discovered frequently in the methadone weaning schedules and have not been described in previous literature. In most standardized protocols for long-term opioid users, ${ }^{3,11,1,5,22,23}$ dose reduction has typically started 1 or 2 days after conversion of opioids to methadone.

It is possible and even likely that the use of 4 phases in this clinical context extended the duration of methadone weaning relative to standardized protocols. Regardless, many of the prospective studies evaluating different protocols have excluded patients with complicating factors, such as long-term mechanical ventilation and central nervous system injuries, ${ }^{2,3,22}$ whereas the current study included such patients. Even with stringent 
inclusion criteria, prospective methadone weaning studies using standardized protocols have had variable success (between $60 \%$ and $80 \%)^{3.22}$

Although standard weaning protocols are assumed to be effective, it can be argued that they often do not take into account the very real challenges presented by patients with complicating factors, many of whom are unsuited to rapid weaning. For instance, in a retrospective study at BC Children's Hospital, Jeffries and others ${ }^{23}$ discovered that for none of the 43 patients included in the study had the hospital's established methadone weaning protocol been followed to completion. This finding illustrates that following a strict methadone regimen is not always effective or feasible and could explain why management of opioid withdrawal at CHEO deviated from literature guidelines for methadone weaning.

One area of interest was the use of a loading phase to reach therapeutic concentrations of methadone in the plasma. For the 30 patients in this study, the loading phase was the shortest phase, with the least variation in duration. Unlike the other 3 phases, the duration of the loading phase was independent of the duration of the overall weaning period. Additionally, the ratio between loading dose and maintenance dose for patients who received both showed high consistency, despite the small sample size $(n=18)$. Consistency in both duration and dose during the loading phase, independent of all other patient factors, suggests that this phase can be somewhat standardized for all patients. The results of this study encouraged a daily loading dose that is 2-fold higher than the desired maintenance dose administered for $48 \mathrm{~h}$.

The choice to analyze patients according to the duration of the tapering phase was based on a number of considerations. First, the tapering phase was the only phase observed for all patients, and it appeared to follow a consistently linear decreasing trend. Furthermore, because of variability in the remaining 3 phases, the tapering phase represented a more practical starting point for determining the actual dose reduction per day and the speed of weaning.

The literature review revealed that certain institutions have used 5-day and 10-day weaning protocols, depending on the duration of continuous morphine infusion. ${ }^{15,23}$ By separating patients into groups based on duration of the tapering phase (see Table 5) and examining each of these groups individually, regression analysis revealed higher $R^{2}$ values (close to the 0.8 threshold for statistical significance) and a more reliable model for estimating daily dose reduction. If the patient who had a 30-day tapering phase (much longer than the criterion for the long-duration weaning group) is excluded from the longduration group, the $R^{2}$ value increases from 0.68 to 0.78 . However, it is difficult to denote outliers in a small group of 13 patients.

Apart from the daily dose reduction and the duration of the tapering phase, none of the clinical factors recorded in this study differed noticeably among the short-, medium-, and long-duration weaning groups. Hence, there was no apparent rationale for favouring one weaning approach over another. More rigorous analysis or studies with broader inclusion criteria will be required to elucidate this matter.

Two variables that were not recorded in this study may explain differences in weaning styles: duration of continuous opioid infusion and withdrawal events. In the literature, longer weaning schedules are typically used following longer periods of continuous opioid infusion. ${ }^{12,23}$ It was initially assumed that recording details for 7 days of opioid dosing would be sufficient for data analysis, and that higher daily opioid doses would be representative of longer infusion duration because of the tolerance effect. However, with a small sample size, opioid dosing is likely insufficient to predict the duration of continuous opioid infusion. Withdrawal events had to be excluded from analysis because of lack of an objective scale, but these are an important consideration for clinicians who prescribe methadone for weaning. Further studies incorporating data for WAT-1 scores (or another objective tool to quantify withdrawal symptoms) and duration of opioid infusion will be required to determine indications for the use of different weaning approaches in pediatrics.

In this study, all but one of the patients were taking at least one medication thought to interact with methadone dosing. More specifically, all but 2 of the patients were taking clonidine and/or benzodiazepines during their weaning. Although it appears that concomitant administration of clonidine, benzodiazepines, and similar medications were appropriately managed in this study population, safety outcomes, adverse events, and withdrawal events were not captured. Therefore, no firm conclusions can be drawn about the safety aspects of concomitant administration of sedative medications with methadone, especially given the diverse study population and the number of complicating medical conditions. Moreover, no relationship was observed between the type or number of concomitant medications and methadone or opioid dosing during weaning (data not shown). We anticipated a correlation between opioid or methadone dosing and clonidine, as clonidine has been shown to decrease postoperative opioid requirements in adults. ${ }^{24}$ However, it was difficult to identify such a correlation in the current study, as the number of patients receiving clonidine $(n=23)$ was far higher than the number of patients not receiving clonidine $(n=7)$, and most of these 23 patients received more than one concomitant medication during their methadone wean.

Mechanical ventilation was a significant complicating factor in this study. One-third of the patients started methadone weaning before extubation, and it was discovered that intubation during methadone weaning significantly increased the duration of both the overlap and the maintenance 
phases. Intubated patients also received higher methadone doses during the overlap phase and higher opioid doses on the day before starting methadone weaning. It is likely that methadone dosing was significantly higher during intubation either because of its sedative properties or to prevent the appearance of opioid withdrawal symptoms during mechanical ventilation; hence, methadone tapering doses could not be administered until after extubation (which would delay the tapering phase). As it stands, all weaning protocols included a loading phase and a tapering phase. Our recommendation is to add overlap and maintenance phases for intubated patients, both to minimize withdrawal symptoms and to ensure proper sedation before transitioning to a standardized methadone maintenance protocol.

Ten $(33 \%)$ of the patients in this study underwent extubation within 7 days before the first dose of methadone. It is unclear whether patients who commenced methadone weaning before extubation had better outcomes, but a recent study of adults in Brazil concluded that enteral methadone can decrease weaning time from mechanical ventilation and ultimately shorten hospital stays. ${ }^{25}$ Future consideration should be given to investigating the benefits of enteral methadone for weaning from mechanical ventilation in the pediatric setting, with the intent of standardizing the process.

This study had limitations due to its retrospective design. The selection criteria limited inclusion to patients whose weaning was completed at the authors' institution, which reduced the number of eligible patients. Unsuccessful weaning represented a large amount of data (given that 32 patients were excluded because weaning was incomplete); dosing patterns might have been clearer if these patients had been included. In addition, patients who completed weaning outside of CHEO were not included, as there was no reliable method of determining the rate and duration of methadone weaning if not documented in the hospital record. Furthermore, the lack of consistent and validated assessment for withdrawal symptoms made it difficult to analyze the indications and rationale for methadone dosing schedules. Without documented assessment of withdrawal symptoms, it was not possible to compare the success of weaning protocols between patients. Finally, the use of concomitant sedative medications during methadone weaning was not standardized at this institution. In particular, safety outcomes based on individual treatment regimens were not captured in this study. Standardized use of medications might have allowed more consistent methadone dosing and facilitated comparisons of concomitant medication administration during methadone use. This would in turn have provided additional insights into concomitant use of sedative medications, safety profiles of these drugs, and any withdrawal events that might have occurred.

\section{CONCLUSIONS}

All patients included in this study had effective withdrawal from opioids without further withdrawal symptoms. Four methadone weaning phases were identified: overlap, loading, maintenance, and tapering. Three durations of tapering were identified: short, medium, and long.

Currently, all weaning protocols at the study hospital include a loading phase and a tapering phase. The addition of overlap and maintenance phases is recommended for intubated patients, to minimize withdrawal symptoms and to ensure proper sedation before transition to a standardized methadone maintenance protocol.

Further studies using this 4- phase, 3-duration approach may confirm the findings of this study.

\section{References}

1. Sorce LR. Adverse responses: sedation, analgesia and neuromuscular blocking agents in critically ill children. Crit Care Nurs Clin North Am. 2005;17(4):441-50.

2. Berens RJ, Meyer MT, Mikhailov TA, Colpaert KD, Czarnecki ML, Ghanayem NS, et al. A prospective evaluation of opioid weaning in opioid-dependent pediatric critical care patients. Anesth Analg. 2006;102(4):1045-50.

3. Bowens CD, Thompson JA, Thompson MT, Breitzka RL, Thompson DG, Sheeran PW. A trial of methadone tapering schedules in pediatric intensive care unit patients exposed to prolonged sedative infusions. Pediatr Crit Care Med. 2011;12(5):504-11.

4. Fonsmark L, Rasmussen YH, Carl P. Occurrence of withdrawal in critically ill sedated children. Crit Care Med. 1999;27(1):196-9.

5. Katz R, Kelly HW, His A. Prospective study on the occurrence of withdrawal in critically ill children who receive fentanyl by continuous infusion. Crit Care Med. 1994;22(5):762-7.

6. Ista E, Van Dijk M, Gamel C, Tibboel D, De Hoog M. Withdrawal symptoms in children after long-term administration of sedatives and/or analgesics: a literature review. "Assessment remains troublesome". Intensive Care Med. 2007;33(8):1396-406.

7. French JP, Nocera M. Drug withdrawal symptoms in children after continuous infusions of fentanyl. J Pediatr Nurs. 1994;9(2):107-13.

8. Playfor SD. Analgesia and sedation in critically ill children. Arch Dis Child Educ Pract Ed. 2008;93(3):87-92.

9. Birchley G. Opioid and benzodiazepine withdrawal syndromes in paediatric intensive care unit: a review of recent literature. Nurs Crit Care. 2009;14(1):26-37.

10. Cho HH, O’Connell JP, Cooney MF, Inchiosa MA Jr. Minimizing tolerance and withdrawal to prolonged pediatric sedation: case report and review of the literature. J Intensive Care Med. 2007;22(3):173-9.

11. Siddappa R, Fletcher JE, Heard AM, Kielma D, Cimino M, Heard CM. Methadone dosage for prevention of opioid withdrawal in children. Paediatr Anaesth. 2003;13(9):805-10.

12. Anand KJS, Willson DF, Berger J, Harrison R, Meert KL, Zimmerman J, et al. Tolerance and withdrawal from prolonged opioid use in critically ill children. Pediatrics. 2010;125(5):E1208-25.

13. Gowing L, Ali R, White JM. Opioid antagonists with minimal sedation for opioid withdrawal. Cochrane Database Syst Rev. 2009;(4):CD002021.

14. Kapur BM, Hutson JR, Chibber T, Luk A, Selby P. Methadone: a review of drug-drug and pathophysiological interactions. Crit Rev Clin Lab Sci. 2011;48(4):171-95.

15. Robertson RC, Darsey E, Fortenberry JD, Pettignano R, Hartley G. Evaluation of an opiate-weaning protocol using methadone in pediatric intensive care unit patients. Pediatr Crit Care Med. 2000;1(2):119-23.

16. SickKids drug handbook and formulary: 2009/2010. Toronto (ON): Hospital for Sick Children; 2009. 
17. Franck LS, Scoppettuolo LA, Wypij D, Curley MAQ. Validity and generalizability of the Withdrawl Assessment Tool-1 (WAT-1) for monitoring iatrogenic withdrawal syndrome in pediatric patients. Pain. 2012;153(1):142-8.

18. Yaster M, Maxwell L.Opioid agonists and antagonists. In: Pain in infants, children, and adolescents. Schechter NL, Berde CB, Yaster M, editors. Baltimore (MD): Williams \& Wilkins; 1993. p. 145-71.

19. Berde CB, Beyer JE, Bournaki MC, Levin CR, Sethna NF. Comparison of morphine and methadone for prevention of postoperative pain in 3- to 7-year-old children. J Pediatr. 1991;119(1 Pt 1):136-41.

20. Miser AW, Chayt KJ, Sandlund JT, Cohen PS, Dothage JA, Miser JS. Narcotic withdrawal syndrome in young adults after the therapeutic use of opiates. Am J Dis Child. 1986;140(6):603-4.

21. Tobias JD, Schleien CL, Haun SE. Methadone as treatment for iatrogenic narcotic dependency in pediatric intensive care unit patients. Crit Care Med. 1990;18(11):1292-3.

22. Meyer MM, Berens RJ. Efficacy of an enteral 10-day methadone wean to prevent opioid withdrawal in fentanyl-tolerant pediatric intensive care unit patients. Pediatr Crit Care Med. 2001;2(4):329-33.

23. Jeffries SA, McGloin R, Pitfield AF, Carr RR. Use of methadone for prevention of opioid withdrawal in critically ill children. Can J Hosp Pharm. 2012;65(1):12-8.

24. Delaunay L, Leppert C, Dechaubry V, Levron JC, Liu N, Bonnet F. Epidural clonidine decreases postoperative requirements for epidural fentanyl. Reg Anesth. 1993;18(3):176-80.

25. Wanzuita R, Poli-de-Figueiredo LF, Pfuetzenreiter F, Cavalcanti AB, Westphal GA. Replacement of fentanyl infusion by enteral methadone decreases the weaning time from mechanical ventilation: a randomized controlled trial. Crit Care. 2012;16(2):R49.
Kazim Giby, BSc, is with the Pharmacy Department, Children's Hospital of Eastern Ontario, and the University of Ottawa, Ottawa, Ontario.

Régis Vaillancourt, OMM, CD, BPharm, PharmD, FCSHP, FFIP, is with the Pharmacy Department, Children's Hospital of Eastern Ontario, Ottawa, Ontario.

Nisha Varughese, PharmD, is with the Pharmacy Department, Children's Hospital of Eastern Ontario, Ottawa, Ontario.

Christina Vadeboncoeur, MD, is with the Pediatric Palliative Care Outreach Team, Children's Hospital of Eastern Ontario, Ottawa, Ontario.

Annie Pouliot, PhD, is with the Pharmacy Department, Children's Hospital of Eastern Ontario, Ottawa, Ontario.

Competing interests: For activities outside the scope of the work reported here, Régis Vaillancourt has received grants from MedBuy, the World Health Organization, the International Pharmaceutical Federation, Algonquin College (Ottawa), Sandoz Canada, and Nycomed, as well as payment for lectures from Purdue Frederic. He is an elected member of the Ontario College of Physicians and a surveyor for Accreditation Canada. No other competing interests declared.

\section{Address correspondence to:}

Dr Régis Vaillancourt

Children's Hospital of Eastern Ontario

401 Smyth Road

Ottawa ON K1H 8L1

e-mail: rvaillancourt@cheo.on.ca

\section{CISADL 2013}

\section{Order your copy of the 2013 Canadian Investigational \& Special Access Drug List today! Please use the CSHP order form found online at CSHP.ca to place your order.}

The CISADL is a compilation of drugs that are currently not marketed in Canada and may have Investigational or Special Access status with Health Canada. This list is not endorsed by Health Canada's Special Access Program. For precise information about the regulatory status of a drug, contact the Special Access Program directly. Entries to the list are contributed to by several Drug Information Centers across Canada and by Canadian drug manufacturers. In cases where non-marketed drugs do not appear on the list, foreign references should be consulted. The content of the list includes generic name and strength/concentration, dosage form, pharmacology/therapeutic use, trade and code name, source (manufacturer/distributor), and comments. Available in English only.

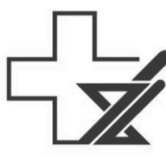
Société canadienne des pharmaciens d'hôpitaux 